Pacific

Journal of

Mathematics

THE SECOND COHOMOLOGY OF SMALL IRREDUCIBLE MODULES FOR SIMPLE ALGEBRAIC GROUPS

George J. MCNinch

Volume $204 \quad$ No. 2

June 2002 


\title{
THE SECOND COHOMOLOGY OF SMALL IRREDUCIBLE MODULES FOR SIMPLE ALGEBRAIC GROUPS
}

\author{
George J. MCNinch
}

Let $G$ be a connected, simply connected, quasisimple algebraic group over an algebraically closed field of characteristic $p>0$, and let $V$ be a rational $G$-module such that $\operatorname{dim} V \leq p$. According to a result of Jantzen, $V$ is completely reducible, and $H^{1}(G, V)=0$. In this paper we show that $H^{2}(G, V)=0$ unless some composition factor of $V$ is a nontrivial Frobenius twist of the adjoint representation of $G$.

\section{Introduction.}

Let $G$ be a quasisimple, connected, simply connected algebraic group over the algebraically closed field $k$ of characteristic $p>0$. By a $G$-module $V$, we always understand a rational $G$-module (one given by a morphism of algebraic groups $G \rightarrow \mathrm{GL}(V)$ ). In this paper, we study the cohomology of a $G$-module $V$ such that $\operatorname{dim} V \leq p$. By results of Jantzen [Jan96] one knows that $V$ is semisimple and that $H^{1}(G, V)=0$.

Recall that the Lie algebra $\mathfrak{g}$ of $G$ is a $G$-module via the adjoint action. Our main result is:

Theorem A. Let $V$ be a $G$-module with $\operatorname{dim} V \leq p$. Then $H^{2}(G, V) \neq 0$ if and only if $V$ has a composition factor isomorphic with a Frobenius twist $\mathfrak{g}^{[d]}$ of $\mathfrak{g}$ for some $d \geq 1$.

Differentiating the representation of $G$ on $V$ gives a representation for the Lie algebra $\mathfrak{g}$ on $V$. Assume that $V^{\mathfrak{g}}=0$. Then the theorem says that $H^{2}(G, V)=0$. For $V$ of this sort, the vanishing of $H^{2}$ is a consequence of the linkage principle for $G$ together with results in Section 2 which give estimates for the dimensions of Weyl modules whose high weights are simultaneously in the low alcove and in the orbit $W_{p} \bullet 0$. In fact, the same argument shows that $H^{i}(G, V)$ is 0 for all $i \geq 1$; see Proposition 5.2. It was pointed out to me that an earlier version of this manuscript contained an overly complicated proof of this observation.

The crucial case for Theorem $\mathrm{A}$ is when $V$ is simple, nontrivial and $V^{\mathfrak{g}}=V$. There is a unique $d \geq 1$ such that the "Frobenius untwist" $V^{[-d]}$ is a $G$-module on which $\mathfrak{g}$ acts nontrivially. We have already seen that 
$H^{i}\left(G, V^{[-d]}\right)=0$ for $i=1,2$, so Theorem A follows from the following two results (see 5.4). [We denote by $h$ the Coxeter number of the group $G$.]

Theorem B. Suppose that $p \geq h$ and that $W$ is a G-module for which $H^{i}(G, W)=0$ for $i=1,2$. Then $H^{2}\left(G, W^{[d]}\right) \simeq \operatorname{Hom}_{G}(\mathfrak{g}, W)$ for all $d \geq 1$.

Theorem C. If $p>h, \operatorname{dim} H^{2}\left(G, \mathfrak{g}^{[d]}\right)=1$ for all $d \geq 1$. For any $p$, there is a $d_{0} \geq 1$ so that $H^{2}\left(G, \mathfrak{g}^{[d]}\right) \neq 0$ for all $d \geq d_{0}$.

Theorem $\mathrm{B}$ is proved in 5.3; it immediately implies the first assertion of Theorem C (see 5.5). We give a proof of the second assertion of Theorem C in Section 5.6.

We end the paper by applying the results of Section 2 to calculations of cohomology groups $H^{i}\left(G_{1}, L\right)$, where $G_{1}$ is the Frobenius kernel, and $L$ is a simple $G_{1}$ module with $\operatorname{dim} L \leq p$; see Proposition 6 .

We make now the following remark concerning our hypothesis on $G$. Suppose that $G$ is quasisimple, but not necessarily simply connected, and let $\pi: G_{\mathrm{sc}} \rightarrow G$ denote the isogeny from the corresponding simply connected covering group. Then any $G$ representation $V$ is also a $G_{\text {sc }}$ representation, and the kernel of $\pi$ is a diagonalizable group scheme. It follows that $\pi$ induces an isomorphism $H^{i}(G, V) \simeq H^{i}\left(G_{\mathrm{sc}}, V\right)$ for each $i \geq 0$; see [CPSvdK77, Remark (2.7)]. I thank W. van der Kallen for pointing this out to me. One may check using Lemma 4.1(A) and Proposition 5.1 that $\operatorname{Lie}\left(G_{\mathrm{Sc}}\right)$ and $\operatorname{Lie}(G)$ are isomorphic simple $G_{\mathrm{sc}}$ representations whenever $\operatorname{dim} G \leq p$. Thus the conclusion of Theorem A remains true for $G$.

We conclude this introduction by remarking that the result of Jantzen [Jan96] cited above is one of several recent results studying the semisimplicity of low dimensional representations of groups in characteristic $p$. See [Ser94], [McN98], [McN99], [Gur99] and [McN00] for related work.

The author would like to acknowledge the hospitality of Bob Guralnick and the University of Southern California during a visit in June 1999; in particular, questions of Guralnick encouraged the author to consider the problems addressed in this paper, and several conversations inspired some useful ideas.

\section{Root systems.}

2.1. We denote by $R$ an indecomposable root system in its weight lattice $X$ with simple roots $S \subset R^{+}$. For each $\alpha \in S$, there is a fundamental dominant weight $\varpi_{\alpha} \in X$; the $\varpi_{\alpha}$ form a $\mathbb{Z}$ basis of $X$.

We write $\alpha_{0}$ for the dominant short root, and $\widetilde{\alpha}$ for the dominant long root in $R$ (these coincide in case there is only one root length).

The Coxeter number of $R$ is given by

$$
h-1=\sup _{\alpha \in R^{+}}\left\{\left\langle\rho, \alpha^{\vee}\right\rangle\right\}=\left\langle\rho, \alpha_{0}^{\vee}\right\rangle .
$$


For $m \in \mathbb{Z}$ and $\alpha \in R$, let $s_{\alpha, m}$ denote the affine reflection of $X_{\mathbb{R}}=X \otimes_{\mathbb{Z}} \mathbb{R}$ in the hyperplane $H_{\alpha, m}=\left\{x \in X_{\mathbb{R}}:\left\langle x, \alpha^{\vee}\right\rangle=m\right\}$.

Let $l>h$ be an integer. The affine Weyl group $W_{l}$ is the group of affine transformations of $X_{\mathbb{R}}$ generated by all $s_{\alpha, l n}$ for $n \in \mathbb{Z}$. According to [Bou72, Ch. VI, §2.1, Prop. 1] $W_{l}$ is isomorphic to the semidirect product of $W$ (the finite Weyl group) with $l \mathbb{Z} R$. The normalizer of $W_{l}$ in the full affine transformation group of $X_{\mathbb{R}}$ contains all translations by $l X$, hence $W_{l}$ is a normal subgroup of $\widehat{W}_{l}$, the semidirect product of $W$ and $l X$. Moreover, $\widehat{W}_{l} / W_{l} \simeq l X / l \mathbb{Z} R \simeq X / \mathbb{Z} R$ is the fundamental group of $R$, which we will denote by $\pi$.

Let $\rho=\frac{1}{2} \sum_{\alpha \in S} \alpha$. We always consider the dot action of $\widehat{W}_{l}$ (also of $W$ and $\left.W_{l}\right)$ on $X$ : For $w \in \widehat{W}_{l}$ and $\lambda \in X$, this is given by $w \bullet \lambda=w(\lambda+\rho)-\rho$.

The closure of the subset $C_{l}$ of $X_{\mathbb{R}}$ given by

$$
C_{l}=\left\{\lambda \in X_{\mathbb{R}} \mid 0<\left\langle\lambda+\rho, \alpha^{\vee}\right\rangle<l \quad \text { for each } \alpha \in R^{+}\right\}
$$

is a fundamental domain for the dot action of $W_{l}$ on $X$; the conjugates of $C_{l}$ under $W_{l}$ are known as alcoves, and $C_{l}$ is the lowest alcove. Since $\widehat{W}_{l}$ normalizes $W_{l}$, [Bou72, Ch. VI, $\S 2.1$ ] shows that $\widehat{W}_{l}$ permutes the alcoves.

Let $\Omega$ be the stabilizer in $\widehat{W}_{l}$ of $C$. Since $W_{l}$ permutes the alcoves simply transitively, one deduces that $\widehat{W}_{l}$ is the semidirect product of $\Omega$ and $W_{l}$. Thus $\Omega \simeq \widehat{W}_{l} / W_{l} \simeq \pi$.

Since $l>h$, the intersection $C_{l} \cap X^{+}$is nonempty. [Note that if $l \leq h$ had been allowed, we would have $C_{l} \cap X^{+}=\{0\}$ in case $l=h$, and $C_{l} \cap X^{+}=\emptyset$ if $l<h$.] It is then clear that $\widehat{W}_{l} \bullet 0 \cap C_{l}=\{\omega \bullet 0 \mid \omega \in \Omega\}$.

2.2. . Let $I$ index the simple roots $S=\left\{\alpha_{i}\right\}$, write $\alpha_{0}^{\vee}=\sum_{i \in I} n_{i} \alpha_{i}^{\vee}$, and put $J=\left\{i \in I \mid n_{i}=1\right\}$. A dominant weight $0 \neq \varpi \in X$ is minuscule if whenever $\lambda \leq \varpi$ and $\lambda$ is a dominant weight, then $\varpi=\lambda$. According to [Bou72, Ch. VI, Exerc. 23, 24], $\varpi$ is minuscule just in case $\varpi=\varpi_{i}$ for some $i \in J$.

For $i \in I \cup\{0\}$, let $S_{i}=S \backslash\left\{\alpha_{i}\right\}$ (so $S_{0}=S$ ). Write $R_{i} \subset R$ for the root subsystem determined by $S_{i}$, and $W_{i}$ for the parabolic subgroup of $W$ associated with $R_{i}$. Let $w_{i} \in W_{i}$ be the unique element which makes all positive roots in $R_{i}$ negative.

For $x \in X$, let $t(x)$ denote the affine translation by $x$; for $i \in J$, let $\gamma_{i}=$ $t\left(l \varpi_{i}\right) w_{0} w_{i} \in \widehat{W}_{l}$. Note that $\gamma_{i}$ represents $\varpi_{i} \in X / \mathbb{Z} R \simeq l X / l \mathbb{Z} R \simeq \widehat{W}_{l} / W_{l}$.

Applying [Bou72, Ch. VI, §2.2 Prop. 6 and Cor.] one obtains:

\section{Proposition.}

(a) Each non-0 coset of $\mathbb{Z} R$ in $X$ is uniquely represented by a minuscule weight. In particular, $|\pi|=|J|+1$. 
(b) The nonidentity elements of $\Omega$ are precisely the $\gamma_{i}$ for $i \in J$. We have

$$
\widehat{W}_{l} \bullet 0 \cap C_{l}=\{0\} \cup\left\{\gamma_{i} \bullet 0=(l-h) \varpi_{i} \mid i \in J\right\} .
$$

2.3. . For a dominant weight $\lambda$, let

$$
d(\lambda)=\prod_{\alpha>0} \frac{\left\langle\lambda+\rho, \alpha^{\vee}\right\rangle}{\left\langle\rho, \alpha^{\vee}\right\rangle}
$$

be the value of Weyl's degree formula at $\lambda$.

Proposition. Let $\lambda=(l-h) \varpi_{i}$ for some $i \in J$.

(a) $d(\lambda) \geq\left(\begin{array}{l}l-1 \\ l-h\end{array}\right)$, with equality if and only if $h-1=\ell\left(w_{0} w_{i}\right)$.

(b) If $l-h \geq 2$ and $h \geq 3$, then $d(\lambda)>l$.

Proof. For $1 \leq k \leq h-1$, let $e(k)$ be the number of $\alpha \in R^{+} \backslash R_{i}^{+}$with $\left\langle\rho, \alpha^{\vee}\right\rangle=k$. The argument in the remark on p. 520-521 of [Ser94] (following Prop. 6) shows that $e(k) \geq 1$ for each $1 \leq k \leq h-1$. Thus, we have

$$
d(\lambda)=\prod_{k=1}^{h-1}\left(\frac{l-h+k}{k}\right)^{e(k)} \geq \prod_{k=1}^{h-1} \frac{l-h+k}{k}=\left(\begin{array}{l}
l-1 \\
l-h
\end{array}\right) .
$$

If $\ell\left(w_{0} w_{i}\right)=\left|R^{+}\right|-\left|R_{i}^{+}\right|=h-1$, then $e(k)=1$ for each $1 \leq k \leq h-1$ and equality holds. This proves (a).

For (b), note that under the given hypothesis we have $l \geq 5$. Since $\left(\begin{array}{l}l-1 \\ l-h\end{array}\right) \geq\left(\begin{array}{c}l-1 \\ 2\end{array}\right)>l$ for all such $l$, (b) follows immediately.

Remark. Using the table in the proof of Proposition 2.4 below, it is straightforward to verify that equality holds in (a) if and only if either $R=A_{r}$ and $i \in\{1, r\}$ or $R=C_{r}$ and $i=1$. (Since $B_{2}=C_{2}$, the latter case includes $B_{2}$ and $i=2$.)

2.4. . In the following, let me emphasize the standing assumption $l>h$.

Proposition. If $0 \neq \lambda \in \widehat{W}_{l} \bullet 0 \cap C$ and $d(\lambda)<l$ then $d(\lambda)=\ell-1$ and $(R, \lambda)$ is listed in the following table. If the rank of $R$ is $\geq 2$, then $l=h+1$.

\begin{tabular}{lll}
$R$ & $l$ & $\lambda$ \\
\hline \hline$A_{1}$ & any & $(l-2) \varpi_{1}$ \\
$A_{l-2}$ & & $\varpi_{1}, \varpi_{l-2}$ \\
$B_{2}$ & $l=5$ & $\varpi_{2}$ \\
$C_{(l-1) / 2}$ & $l$ odd & $\varpi_{1}$
\end{tabular}

Proof. The rank 1 situation leads to the item listed in the table. When the rank is at least 2 , one applies Proposition 2.3 to obtain $l=h+1$, whence $\lambda=\varpi_{i}$ for some $i \in J$; i.e., $\lambda$ is minuscule. 
We handle the minuscule cases by classification. For each indecomposable root system $R$ for which $J \neq \emptyset$, we list in the following table the Coxeter number, the set $J$, and the value $d\left(\varpi_{i}\right)$ for each $i \in J$. The simple roots are indexed as in the tables in [Bou72, Planche I-X]; the data recorded here, with the exception of the values $d\left(\varpi_{i}\right)$, may be verified by inspecting those tables as well. The values $d\left(\varpi_{i}\right)$ are well-known (and can anyway be computed from the formula, or by representation theoretic arguments).

\begin{tabular}{l|lll} 
Type of $R$ & $h$ & $J$ & $d\left(\varpi_{i}\right), i \in J$ \\
\hline \hline$A_{r}$ & $r+1$ & $\{1,2, \ldots, r\}$ & $\left(^{r+1}{ }_{i}\right)$ \\
$B_{r}, r \geq 2$ & $2 r$ & $\{r\}$ & $2^{r}$ \\
$C_{r}, r \geq 2$ & $2 r$ & $\{1\}$ & $2 r$ \\
$D_{r}, r \geq 4$ & $2 r-2$ & $\{1, r-1, r\}$ & $2 r, 2^{r-1}, 2^{r-1}$ respectively \\
$E_{6}$ & 12 & $\{1,6\}$ & 27,27 \\
$E_{7}$ & 18 & $\{7\}$ & 56
\end{tabular}

From this table, one can list all pairs $(R, \lambda)$ for which $R$ has Coxeter number $l-1$ and $\lambda$ is minuscule. It is a simple matter to see that $d(\lambda)<l$ only when $(R, \lambda)$ is as claimed.

\section{The algebraic groups.}

3.1. Let $k$ be an algebraically closed field of characteristic $p>0$, and let $G$ be a connected, simply connected semisimple algebraic $k$-group. The non-0 weights of a maximal torus $T \leq G$ on $\mathfrak{g}=\operatorname{Lie}(G)$ form a root system $R$ of rank $r=\operatorname{dim} T$ in the character group $X=X^{*}(T)$. Since $G$ is simply connected, $X$ identifies with the full weight lattice of $R$ as in Section 2. We fix a choice of simple roots $S$ and positive roots $R^{+}$. The dominant weights are denoted $X^{+}$. The group $G$ is assumed to be quasisimple; i.e., the root system $R$ is indecomposable.

3.2. . For each dominant weight $\lambda \in X^{+}$, the space of global sections of the corresponding line bundle on the flag variety affords an indecomposable rational $G$-module $H^{0}(\lambda)$ with simple socle. The modules $L(\lambda)=\operatorname{soc} H^{0}(\lambda)$ comprise all of the simple rational modules for $G$ (and are pairwise nonisomorphic).

The character of each $H^{0}(\lambda)$ is the same as in characteristic 0 ; hence in particular $\operatorname{dim}_{k} H^{0}(\lambda)$ is given by the Weyl degree formula, whose value at $\lambda$ we denote $d(\lambda)$ as in 2.3 .

3.3. . Any dominant $\lambda$ may be written as a finite sum $\sum_{i \geq 0} p^{i} \lambda_{i}$ with each $\lambda_{i}$ a restricted weight. Recall that a dominant weight $\mu$ is restricted if $\left\langle\mu, \alpha^{\vee}\right\rangle<p$ for all simple roots $\alpha$. Steinberg's tensor product theorem says:

$$
L(\lambda) \simeq L\left(\lambda_{0}\right) \otimes L\left(\lambda_{1}\right)^{[1]} \otimes L\left(\lambda_{2}\right)^{[2]} \otimes \cdots
$$


where for a $G$-module $V, V^{[m]}$ stands for the $m$-th Frobenius twist of $V$.

For $d \geq 1$, let $G_{d}$ be the $d$-th Frobenius kernel of $G$. Let $V$ be a rational $G$-module and $m \geq 1$. If there is a rational $G$ module $W$ with $W^{[m]} \simeq V$, we regard $W$ as the Frobenius untwist $W=V^{[-m]}$ of $V$. Now regard $V$ as a module for $G_{d}$. Since $G_{d}$ is a normal subgroup scheme, $G$ acts on $V^{G_{d}}$; since $G_{d}$ acts trivially on this $G$-module, there is an untwisted rational $G$-module $\left(V^{G_{d}}\right)^{[-d]}$. It follows that there is an untwist $H^{i}\left(G_{d}, V\right)^{[-d]}$ for all $i \geq 0$.

Consider now two $G$-modules $V_{1}$ and $V_{2}$, and form $W=V_{1} \otimes V_{2}^{[\bar{d}]}$. The Frobenius kernel $G_{d}$ acts trivially on $V_{2}^{[d]}$, so that

$$
H^{i}\left(G_{d}, W\right)^{[-d]} \simeq H^{i}\left(G_{d}, V_{1}\right)^{[-d]} \otimes V_{2}
$$

as $G$-modules for every $i \geq 0$.

3.4. . Let $W_{p} \leq \widehat{W}_{p}$ be as in Section 2 (for $l=p$ ), let $C=C_{p} \cap X^{+}$denote the dominant weights in the lowest alcove, and let $\bar{C}=\bar{C}_{p} \cap X^{+}\left(\bar{C}_{p}\right.$ is the closure in $X_{\mathbb{R}}$ ).

Proposition. Let $\lambda \in X^{+}$.

(a) If $H^{i}(G, L(\lambda)) \neq 0$ for some $i \geq 0$, then $\lambda \in W_{p} \bullet 0$.

(b) If $H^{i}\left(G_{1}, L(\lambda)\right) \neq 0$ for some $i \geq 0$, then $\lambda \in \widehat{W_{p}} \bullet 0$.

(c) $H^{i}\left(G, H^{0}(\lambda)\right)=0$ for all $i>0$.

(d) If $\lambda \in \bar{C}$, then $L(\lambda)=H^{0}(\lambda)$; in particular, $\operatorname{dim} L(\lambda)=d(\lambda)$.

Proof. (a) follows from the linkage principle for $G$ [Jan87, Cor. II.6.17], and (b) from the linkage principle for $G_{1}$ [Jan87, Lemma II.9.16]. (c) follows from [Jan87, II.4.12]. (d) follows from [Jan87, II.6.13, II.5.10].

\section{The Lie algebra and the cohomology of $G_{1}$.}

We want to describe explicitly the cohomology $H^{*}\left(G_{1}, k\right)$ in degree $\leq 2$. For this, we need some information on the Lie algebra $\mathfrak{g}$.

4.1. . Recall that the prime $p$ is $b a d[=$ not good $]$ for the indecomposable root system $R$ if one of the following holds: $p=2$ and $R$ is not of type $A_{r}$; $p=3$ and $R$ is of type $G_{2}, F_{4}$, or $E_{r} ; p=5$ and $R$ is of type $E_{8}$.

The prime $p$ is very good if it is not bad, and in case $R=A_{r}$, if also $p$ does not divide $r+1$. Notice that if $p>h$, then $p$ is very good.

Application of the summary in [Hum95, 0.13] yields the following:

Lemma A. Assume that $p$ is very good. Then $\mathfrak{g}$ is a simple Lie algebra. The adjoint $G$-module is simple, self-dual, and isomorphic with $L(\widetilde{\alpha})$ where $\widetilde{\alpha}$ is the dominant long root.

Lemma B. Assume that $p \geq h$. If $W$ is any $G$-module, then $\operatorname{Hom}_{G}\left(\mathfrak{g}, W^{[d]}\right)$ $=0$ for $d \geq 1$. 
Proof. When $p>h$ this follows since by the previous lemma $\mathfrak{g}$ is a simple $\mathfrak{g}$-module with restricted highest weight. When $p=h$, we have $R=A_{p-1}$. Since $G$ is simply connected, we have $\mathfrak{g}=\mathfrak{s l}_{p}$. Thus $\mathfrak{g}$ is an indecomposable $G$-module with unique simple quotient $L(\widetilde{\alpha})$, and the lemma follows.

4.2. . Let $B$ be a Borel subgroup of $G$, and let $\mathfrak{u}$ be the nilradical of $\operatorname{Lie}(B)$. Regarding $\mathfrak{u}^{*}$ as a $B$-module, we get a vector bundle on $G / B$ which we also write as $\mathfrak{u}^{*}$. According to $[\mathbf{A J} 84,3.8]$, the formal character of the $G$-module $H^{0}\left(G / B, \mathfrak{u}^{*}\right)$ is $\chi(\widetilde{\alpha})=\operatorname{ch}\left(\mathfrak{g}^{*}\right)$.

Let $\mathcal{N} \subset \mathfrak{g}$ be the nilpotent cone. There is by [AJ84, 3.9] an injective homomorphism of graded algebras $k[\mathcal{N}] \rightarrow H^{0}\left(G / B, S \mathfrak{u}^{*}\right)$.

Lemma. For simply connected, quasisimple algebraic groups $G, \mathfrak{g}^{*} \simeq k[\mathcal{N}]_{1}$ $\simeq H^{0}\left(G / B, \mathfrak{u}^{*}\right)$.

Proof. Let $I(\mathcal{N}) \triangleleft k[\mathfrak{g}]=S \mathfrak{g}^{*}$ be the (homogeneous) defining ideal of the variety $\mathcal{N}$. We need to show that $I(\mathcal{N})_{1}=0$. If not, then $\mathcal{N} \subset V \subset \mathfrak{g}$ for some proper $G$-submodule $V$. A look at the summary in [Hum95, 0.13] shows that, since $G$ is simply connected, the only $G$-submodules of $\mathfrak{g}$ have dimension 0 or 1 . On the other hand, by [Hum95, Theorem 6.19], the variety $\mathcal{N}$ has codimension $\operatorname{rank}(G)$ in $\mathfrak{g}$ and so clearly can't be contained in a 1 dimensional linear subspace!

\section{Remarks.}

(1) Here is a fancier result which implies the lemma if we assume that the prime $p$ is good for $G$. Since $G$ is simply connected and $p$ is good, the Springer resolution

$$
\varphi: \widetilde{\mathcal{N}}=G \times^{B} \mathfrak{u} \rightarrow \mathcal{N}
$$

given by $(g, X) \mapsto \operatorname{Ad}(g)(X)$ is a desingularization, hence in particular a birational map; see [Hum95, Theorem 6.3 and Theorem 6.20]. Again since $G$ is simply connected and $p$ is good, the variety $\mathcal{N}$ is normal ([Hum95, Theorem 4.24]). Standard arguments then yield an isomorphism of graded algebras $k[\mathcal{N}] \stackrel{\varphi^{*}}{\simeq} \Gamma\left(\widetilde{\mathcal{N}}, \mathcal{O}_{\widetilde{\mathcal{N}}}\right)$. Finally, the projection $\widetilde{\mathcal{N}} \rightarrow G / B$ is an affine morphism, so that $\Gamma\left(\widetilde{\mathcal{N}}, \mathcal{O}_{\widetilde{\mathcal{N}}}\right)=H^{0}\left(G / B, S \mathfrak{u}^{*}\right)$ as a graded algebra.

(2) On the other hand, if $G=P G L_{r}$, and $p \mid r$, one can find a linear form on $\mathfrak{g}$ that vanishes on $\mathcal{N}$, hence there can be no isomorphism $k[\mathcal{N}]_{1} \rightarrow H^{0}\left(G / B, \mathfrak{u}^{*}\right)$ (compare formal characters). So the lemma can fail when $G$ is not simply connected. [Note that $\varphi$ is not birational in this example. One can show that there is a $G_{s c}$-isomorphism $\psi: \widetilde{\mathcal{N}}_{\mathrm{sc}} \rightarrow$ $\widetilde{\mathcal{N}}$ (using some obvious notations). We get therefore a commuting 
diagram:

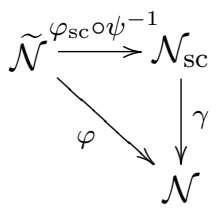

The map $\varphi_{\text {sc }} \circ \psi^{-1}$ is birational. Since $\gamma^{*} k(\mathcal{N}) \subset k\left(\mathcal{N}_{s c}\right)$ is a proper purely inseparable extension, so too is $\varphi^{*} k(\mathcal{N}) \subset k(\widetilde{\mathcal{N}})$.]

\section{Proposition.}

(1) If $p \neq 2$ or if $R$ is not of type $C_{r}$, then $H^{1}\left(G_{1}, k\right)=0$.

(2) Assume that $p \geq h$. Then $H^{2}\left(G_{1}, k\right)^{[-1]} \simeq \mathfrak{g}^{*}$ as $G$-modules.

Proof. For (1) see [Jan87, Lemma II.12.1]. For (2), first suppose that $p>h$. By $[\mathbf{A J} 84,3.7,3.9]$, there is a $G$-equivariant isomorphism of graded rings $k[\mathcal{N}]^{\prime} \simeq H^{*}\left(G_{1}, k\right)^{[-1]}$ where $k[\mathcal{N}]^{\prime}$ is again the graded coordinate ring of $\mathcal{N}$, but with the linear functions on $\mathfrak{g}$ given degree 2 . The claim now follows from the lemma.

When $p=h$, apply [AJ84, Cor. 6.3] to see that $H^{2}\left(G_{1}, k\right)^{[-1]} \simeq$ $H^{0}\left(G / B, \mathfrak{u}^{*}\right)$; the claim follows again from the lemma in this case.

\section{Low dimensional modules for $G$.}

5.1. . We recall first some facts about low dimensional modules established in [Jan96] and [Ser94].

Proposition. Let $L$ be a simple nontrivial restricted $G$ module with highest weight $\lambda$. Suppose that $\operatorname{dim} L \leq p$.

(a) $\lambda \in \bar{C}$.

(b) $\lambda \in C$ if and only if $\operatorname{dim}_{k} L<p$.

(c) $h \leq p$. If moreover $\operatorname{dim} L<p$, then $h<p$.

(d) If $R$ is not of type $A$ and $\operatorname{dim} L=p$, then $h<p$. If $p=h$ and $\operatorname{dim} L=p$, then $R=A_{p-1}$ and $\lambda=\varpi_{i}$ with $i \in\{1, p-1\}$.

Proof. (a) follows from [Jan96, Lemma 1.4], and (b) from [Jan96, 1.6], see also [Ser94]. For (c), note first that (a) implies $\operatorname{dim} L=d(\lambda)$ by Proposition 3.4(d). If $\lambda \in \bar{C} \backslash C$, then (a) and (b) imply that $\operatorname{dim} L=p$, whence $p=h$ follows from Weyl's degree formula. (c) now follows since $C$ is empty if $p<h$ and $C=\{0\}$ if $p=h$.

In [Jan96, 1.6], Jantzen made a list of all simple restricted modules for $G$ with dimension $p$. Inspecting that list yields (d).

5.2. Vanishing results when $\mathfrak{g}$ acts nontrivially. Let $L$ be a simple module for $G$. 
Proposition. If $G_{1}$ (equivalently: $\mathfrak{g}$ ) acts nontrivially on $L$ and $\operatorname{dim} L \leq p$, then $H^{i}(G, L)=0$ for all $i \geq 0$.

Proof. Write the highest weight of $L$ as $\lambda=\mu_{1}+p \mu_{2}$ with $\mu_{1}$ restricted. Since $L^{\mathfrak{g}}=0$, we have $\mu_{1} \neq 0$. Since $p \geq \operatorname{dim} L \geq \operatorname{dim} L\left(\mu_{1}\right)$, Proposition 5.1 implies that $\mu_{1} \in \bar{C}$ and that $h \leq p$. We have in particular that $L\left(\mu_{1}\right)=$ $H^{0}\left(\mu_{1}\right)$, hence the proposition will follow from Proposition 3.4 if we show that $\mu_{2}$ is 0 .

If $\operatorname{dim} L=p$, Steinberg's tensor product theorem gives $\mu_{2}=0$. If $\operatorname{dim} L<$ $p$ then 5.1 shows that $p<h$ and $\mu_{1} \in C$. If $H^{i}(G, L) \neq 0$ for some $i$, then $\lambda \in W_{p} \bullet 0$ by the linkage principle, whence $\mu_{1} \in W \bullet 0+p X=\widehat{W}_{p} \bullet 0$. Now Proposition 2.4 applies; it shows that $\operatorname{dim} L\left(\mu_{1}\right)=p-1$ whence we have $\mu_{2}=0$ by another application of Steinberg's theorem.

5.3. Second cohomology. Here we prove our main tool for describing second cohomology; first we require the following:

Lemma. Let $E_{2}^{p, q} \Longrightarrow H^{p+q}$ be a convergent, first quadrant spectral sequence.

(1) If $E_{2}^{0,1}=E_{2}^{1,1}=E_{2}^{0,2}=0$, then $H^{2} \simeq E_{2}^{2,0}$.

(2) If $E_{2}^{1,0}=E_{2}^{1,1}=E_{2}^{2,0}=0$, then $H^{2} \simeq E_{2}^{0,2}$.

Proof. We verify (1), the argument for (2) is the same. We must show that $E_{\infty}^{2,0} \simeq E_{2}^{2,0}$; first note that $E_{3}^{2,0}$ is the cohomology of the sequence

$$
E_{2}^{0,1} \rightarrow E_{2}^{2,0} \rightarrow E_{2}^{4,-1}
$$

from which we get $E_{3}^{2,0} \simeq E_{2}^{2,0}$. For any first quadrant spectral sequence one has (by similar reasoning) that $E_{a}^{2,0} \simeq E_{a+1}^{2,0}$ for $a>2$, so we get the desired isomorphism.

Theorem. Suppose that $p \geq h$. Let $V$ be a $G$-module for which $H^{i}(G, V)=$ 0 for $i=1,2$, and let $d \geq 1$.

(1) $H^{1}\left(G, V^{[d]}\right)=0$, and

(2) $H^{2}\left(G, V^{[d]}\right) \simeq \operatorname{Hom}_{G}(\mathfrak{g}, V)$.

Proof. The Frobenius kernel $G_{1}$ is a normal subgroup of $G$; thus there is a Lyndon-Hochschild-Serre spectral sequence computing $H^{i}\left(G, V^{[d]}\right)$ which in view of 3.3 (1) has the form

$$
E_{2}^{s, t}=H^{s}\left(G, H^{t}\left(G_{1}, V^{[d]}\right)^{[-1]}\right)=H^{s}\left(G, H^{t}\left(G_{1}, k\right)^{[-1]} \otimes V^{[d-1]}\right) .
$$

If $t=1, E_{2}^{s, t}=0$ by Proposition $4.2(1)$.

There is an exact sequence of the form [Jan87, I.4.1(4)]

$$
0 \rightarrow E_{2}^{1,0} \rightarrow H^{1}\left(G, V^{[d]}\right) \rightarrow E_{2}^{0,1}=0 .
$$


Thus $H^{1}\left(G, V^{[d]}\right) \simeq E_{2}^{1,0} \simeq H^{1}\left(G, V^{[d-1]}\right)$. We get now (1) by induction on $d$.

Proposition $4.2(2)$ shows now that $H^{2}\left(G_{1}, k\right) \simeq \mathfrak{g}^{*}$. Thus, the only possible non- $0 E_{2}$ terms of total degree 2 are

$$
\begin{aligned}
& E_{2}^{0,2}=H^{0}\left(G, \mathfrak{g}^{*} \otimes V^{[d-1]}\right)=\operatorname{Hom}_{G}\left(\mathfrak{g}, V^{[d-1]}\right) \\
& E_{2}^{2,0}=H^{2}\left(G, V^{[d-1]}\right) .
\end{aligned}
$$

For $d>1$, we apply Lemma 4.1(B) to see that $E_{2}^{0,2}=0$ whence $H^{2}\left(G, V^{[d]}\right)$ $\simeq E_{2}^{2,0}=H^{2}\left(G, V^{[d-1]}\right)$ by part (1) of the lemma; thus (2) will follow provided it holds for $d=1$. In that case, we have $E_{2}^{2,0}=0$ by assumption, and the result just proved in part (1) shows that $E_{2}^{1,0}=0$. Thus part (2) of the lemma applies; it shows that $H^{2}\left(G, V^{[1]}\right) \simeq E_{2}^{0,2}=\operatorname{Hom}_{G}(\mathfrak{g}, V)$ as desired.

5.4. The second cohomology of small modules. Let $L=L(\lambda)$ be a simple $G$-module, and suppose that $\operatorname{dim} L \leq p$. Proposition 5.2 showed that the vanishing of cohomology for $L$ is a consequence of the linkage principle when $\lambda \notin p X$. However, if $\lambda \in p \mathbb{Z} R, \lambda$ is linked to 0 , so the linkage principle does not yield vanishing. The following result shows that, despite the linkage of $\lambda$ and 0 in this case, the second cohomology is usually 0 .

Theorem. Let $L$ be a simple $G$-module with $\operatorname{dim} L \leq p$. If $H^{2}(G, L) \neq 0$, then $L \simeq \mathfrak{g}^{[d]}$ for some $d \geq 1$.

Proof. Let $L^{\prime}$ be such that $L \simeq\left(L^{\prime}\right)^{[d]}$ for $d \geq 0$, and such that $\mathfrak{g}$ acts nontrivially on $L^{\prime}$. We have by 5.1 that $p \geq h$. Also, we have by Proposition 5.2 that $H^{i}\left(G, L^{\prime}\right)=0$ for $i \geq 1$. If $d=0$, we are done. If $d>1$, then Theorem 5.3 applies, and we get that

$$
H^{2}(G, L) \simeq \operatorname{Hom}_{G}\left(\mathfrak{g}, L^{\prime}\right) .
$$

We get by Proposition 5.1 that $p>h$ unless $R=A_{p-1}$ and $L^{\prime}=L\left(\varpi_{i}\right)$ with $i \in\{1, p-1\}$. If $p>h$, then $\mathfrak{g}$ is a simple $G$-module by Lemma 4.1(A). So if $\operatorname{Hom}_{G}\left(\mathfrak{g}, L^{\prime}\right) \neq 0$ then $L^{\prime} \simeq \mathfrak{g}$ whence $L \simeq \mathfrak{g}^{[d]}$ as claimed.

In the remaining case, one must just note that weight considerations yield $\operatorname{Hom}_{G}\left(\mathfrak{g}, L\left(\varpi_{i}\right)\right)=0$ for $i=1, p-1$, whence $H^{2}(G, L)=0$.

5.5. The second cohomology of twists of the adjoint module. The first assertion of Theorem $\mathrm{C}$ of the introduction follows from the following:

Proposition. Assume that $p>h$. Then $H^{1}\left(G, \mathfrak{g}^{[d]}\right)=0$ and $H^{2}\left(G, \mathfrak{g}^{[d]}\right) \simeq$ $\operatorname{End}_{G}(\mathfrak{g})$ has dimension 1 for $d \geq 1$.

Proof. Since $p>h$, Lemma 4.1(A) shows that $\mathfrak{g}$ is the simple module with highest weight $\widetilde{\alpha}$. It follows that $\mathfrak{g}=H^{0}(\widetilde{\alpha})$, and thus that $H^{i}(G, \mathfrak{g})=0$ for $i \geq 1$ by Proposition 3.4. The proposition now follows from Theorem 5.3. 
Remark. Note that $\operatorname{dim} \mathfrak{g}>h$ (in fact, $\operatorname{dim} \mathfrak{g}=(h+1) r$ where $r$ is the rank of $G)$. So we get also: If $\operatorname{dim} \mathfrak{g} \leq p$, then $\operatorname{dim} H^{2}\left(G, \mathfrak{g}^{[d]}\right)=1$ for $d \geq 1$.

5.6. A second proof. Here we give a second proof of the non-vanishing of $H^{2}$ for twists of the adjoint module; the result proved here verifies the remaining assertion of Theorem $\mathrm{C}$ of the introduction. We have included the argument since it offers some "explanation" for the non-vanishing.

The group $G$ arises by base change from a split reductive group scheme $\mathbf{G}$ over $\mathbb{Z}$. Let $\mathbb{Z}_{p}$ be the complete ring of $p$-adic integers, and let $\mathbb{Q}_{p}$ be its field of quotients. For any finite field extension $F$ of $\mathbb{Q}_{p}$, let $\mathfrak{o}$ denote the integers in $F$. The residue field $\mathfrak{o} / \mathfrak{m}$ may be identified with the extension $\mathbb{F}_{q}$ of $\mathbb{F}_{p}$.

Let $K$ denote the group of points $\mathbf{G}(\mathfrak{o})$ regarded as a subgroup of $\mathbf{G}(F)$. Since $\mathbf{G}$ is smooth, the reduction homomorphism $K \rightarrow \mathbf{G}\left(\mathbb{F}_{q}\right)$ is surjective (see [Tit79, 3.4.4]).

For $n \geq 1$, let $K_{n} \subset K$ be the kernel of the map $K \rightarrow \mathbf{G}\left(\mathfrak{o} / \mathfrak{m}^{n}\right)$. Note that $K / K_{1}=\mathbf{G}\left(\mathbb{F}_{q}\right)$ acts by conjugation on each quotient $K_{n} / K_{n+1}$.

Proposition. (a) There is for each $m \geq 1$ a canonical isomorphism $K_{m} / K_{m+1} \simeq \mathfrak{g}_{\mathbb{F}_{q}}$ as representations for $\mathbf{G}\left(\mathbb{F}_{q}\right)$, where $\mathfrak{g}_{\mathbb{F}_{q}}$ is the Lie algebra of $\mathbf{G}_{\mathbb{F}_{q}}$.

(b) If $H^{2}\left(\mathbf{G}\left(\mathbb{F}_{q}\right), \mathfrak{g}_{\mathbb{F}_{q}}\right)=0$, the exact sequence of groups

$$
1 \rightarrow K_{1} \rightarrow K \rightarrow \mathbf{G}\left(\mathbb{F}_{q}\right) \rightarrow 1
$$

splits.

(c) There is a p-power $q_{0}$, depending only on the root system $R$ of $G$, such that $H^{2}\left(\mathbf{G}\left(\mathbb{F}_{q}\right), \mathfrak{g}_{\mathbb{F}_{q}}\right) \neq 0$ whenever $q \geq q_{0}$.

(d) There is an integer $a_{0} \geq 1$ such that $H^{2}\left(G, \mathfrak{g}^{[a]}\right) \neq 0$ whenever $a \geq a_{0}$.

Proof. (a) Follows from [DG70, II.§4.3]. (b) Since $K_{1}$ is a pro- $p$ group [PR94, Lemma 3.8], this follows from [Ser67, Lemma 3].

(c) Choose a $\mathbb{Q}_{p}$ vectorspace $V$ and a nontrivial faithful $\mathbb{Q}_{p}$-rational representation $\mathbf{G}_{\mathbb{Q}_{p}} \rightarrow \mathrm{GL}(V)$. For each extension $F$ of $\mathbb{Q}_{p}$ with integers $\mathfrak{o}$, the group $K=\mathbf{G}(\mathfrak{o})$ is a subgroup of (the group of $F$-points of) $\mathrm{GL}\left(V_{F}\right)$. If $H^{2}\left(\mathbf{G}\left(\mathbb{F}_{q}\right), \mathfrak{g}_{\mathbb{F}_{q}}\right)=0$, the sequence in (b) is split and $V_{F}$ is a nontrivial $F\left[\mathbf{G}\left(\mathbb{F}_{q}\right)\right]$-module.

Since $F$ has characteristic 0 , it is well-known that the minimal dimension of a nontrivial $F\left[\mathbf{G}\left(\mathbb{F}_{q}\right)\right]$ module is bounded below by the value $f(q)$ of a polynomial $f \in \mathbb{Q}[x]$, depending only on $G$, for which $f(q) \rightarrow \infty$ as $q \rightarrow \infty$. We may choose $q_{0}$ such that $f(q)>\operatorname{dim}_{\mathbb{Q}_{p}} V$ for each $q>q_{0}$, and (c) follows at once.

(d) now follows from (c) and [CPSvdK77, Cor. 6.9]. 


\section{Small simple modules for $G_{1}$.}

Combining results of [KLT99] with the results recorded in 2.4, we obtain some explicit results on $G_{1}$ cohomology of low dimensional simple modules:

Proposition. Let $L$ be a nontrivial simple $G_{1}$ module with $\operatorname{dim} \leq p$. Assume for some $i \geq 0$ that $H^{i}\left(G_{1}, L\right) \neq 0$. Then $\operatorname{dim} L=p-1$. Moreover, there is a quadruple $(R, \lambda, i(0), V)$ in the following table for which $R$ is the root system of $G, \lambda$ the high weight of $L, i \geq i(0)$ and $H^{i(0)}\left(G_{1}, L\right)^{[-1]} \simeq V$ as $G$-modules.

\begin{tabular}{|c|c|c|c|}
\hline$R$ & $\lambda$ & $i(0)$ & $H^{i(0)}\left(G_{1}, L\right)^{[-1]}$ \\
\hline 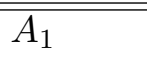 & $\overline{(p-2) \varpi_{1}}$ & 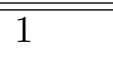 & 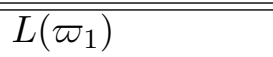 \\
\hline$A_{p-2}$ & $\varpi_{1}, \varpi_{p-2}$ & $p-2$ & $L(\lambda)$ \\
\hline$C_{(p-1) / 2}$ & $\varpi_{1}$ & $p-2$ & $L(\lambda)$ \\
\hline
\end{tabular}

Proof. By [Jan87, Prop. II.3.14], $L=\operatorname{res}_{G_{1}}^{G} L(\lambda)$ for some restricted dominant weight $0 \neq \lambda$. Thus $L(\lambda)$ is a restricted, simple $G$ module with dimension $\leq p$. It follows from Proposition 5.1 that $h \leq p$, that $\lambda \in \bar{C}$, and that $L=H^{0}(\lambda)$ as modules for $G$.

Suppose that $H^{i}\left(G_{1}, L\right) \neq 0$ for some $i$. By the linkage principle for $G_{1}$ (Proposition 3.4(b)), we must have $\lambda \in \widehat{W_{p}} \bullet 0$, hence $\lambda \in C$. This implies that $h<p$. Proposition 2.2 shows that $\lambda=(p-h) \varpi_{i}=w_{0} w_{i} \bullet 0+p \varpi_{i}$ for some $i \in J$, and Proposition 2.3 yields $\operatorname{dim} L=p-1$ and lists the possible pairs $(R, \lambda)$.

For $h<p$, Kumar, Lauritzen and Thomsen [KLT99, Theorem 8] have extended a result of Andersen and Jantzen [AJ84, 3.7]; this result implies in particular that the minimal degree for which $H^{*}\left(G_{1}, L\right)$ is non-0 is $\ell\left(w_{0} w_{i}\right)$, and that

$$
H^{\ell\left(w_{0} w_{i}\right)}\left(G_{1}, L\right)^{[-1]} \simeq H^{0}\left(\varpi_{i}\right) .
$$

It is straightforward to compute for each pair $(R, \lambda)$ the length $\ell\left(w_{0} w_{i}\right)$; one gets in this way the result.

Remark. The Theorem implies the fact (used by Jantzen in the proof of [Jan96, Lemma 1.7]) that $H^{1}\left(G_{1}, L\right)=0$ for all simple $G_{1}$ modules $L$ with $\operatorname{dim} L \leq p$. The argument used by Jantzen there relied on the calculations of $H^{1}$ carried out in [Jan91].

\section{References}

[AJ84] H.H. Andersen and J.C. Jantzen, Cohomology of induced representations for algebraic groups, Math. Ann., 269 (1984), 487-525, MR 86g:20057, Zbl 0529.20027. 
[Bou72] N. Bourbaki, Groupes et algèbres de Lie, Chapitres 4, 5, 6, Hermann, Paris, 1972, MR 58 \#28083a, Zbl 0249.22001.

[CPSvdK77] E. Cline, B. Parshall, L. Scott and W. van der Kallen, Rational and generic cohomology, Invent. Math., 39 (1977), 143-163, MR 55 \#12737, Zbl 0346.20031.

[DG70] M. Demazure and P. Gabriel, Groupes algébriques. Tome I: Géométrie algébrique, généralités, groupes commutatifs, Masson \& Cie, Éditeur, Paris, 1970, Avec un appendice 'Corps de classes local' par Michiel Hazewinkel, MR 46 \#1800, Zbl 0203.23401.

[Gur99] R.M. Guralnick, Small representations are completely reducible, J. Algebra, 220(2) (1999), 531-541, MR 2000m:20018, Zbl 0941.20001.

[Hum95] J.E. Humphreys, Conjugacy classes in semisimple algebraic groups, Math. Surveys and Monographs, 43, Amer. Math. Soc., 1995, MR 97i:20057, Zbl 0834.20048.

[Jan87] J.C. Jantzen, Representations of algebraic groups, Pure and Applied Mathematics, 131, Academic Press, Orlando, FL, 1987, MR 89c:20001, Zbl 0654.20039.

[Jan91] _ First cohomology groups for classical Lie algebras, Representation Theory of Finite Groups and Finite Dimensional Algebras (Bielefeld) (G.O. Michler and C.M. Ringel, eds.), Progr. in Math., 95, Birkhäuser, Boston, 1991, 289-315, MR 92e:17024, Zbl 0749.17020.

[Jan96] L L L L L dimensional representations of reductive groups are semisimple, Algebraic Groups and Related Subjects; a Volume in Honour of R. W. Richardson (G.I. Lehrer et al., ed.), Austral. Math. Soc. Lect. Ser., Cambridge Univ. Press, Cambridge, 1996, 255-266, MR 99g:20079, Zbl 0877.20029.

[KLT99] S. Kumar, N. Lauritzen and J.F. Thomsen, Frobenius splitting of cotangent bundles of flag varieties, Invent. Math., 136 (1999), 603-621, MR 2000g:20088, Zbl 0959.14031.

[McN98] G.J. McNinch, Dimensional criteria for semisimplicity of representations, Proc. London Math. Soc., 76(3) (1998), 95-149, MR 99b:20076, Zbl 0891.20032 .

[McN99] S Semisimple modules for finite groups of Lie type, J. London Math. Soc. 60 (1999), no. 2, 771-792, MR 2001k:20096, Zbl 0961.20014.

[McN00] S Semisimplicity of exterior powers of simple representations of groups, J. Alg., 225 (2000), 646-666, MR 2001c:20016, Zbl 0971.20005.

[PR94] V. Platonov and A. Rapinchuk, Algebraic groups and number theory, Pure and Applied Mathematics, 139, Academic Press, 1994, English translation, MR 95b:11039, Zbl 0841.20046.

[Ser67] J.P. Serre, Local class field theory, Algebraic Number Theory (Proc. Instructional Conf., Brighton, 1965), Thompson, Washington, D.C., 1967, 128-161, MR 36 \#3753.

[Ser94] Sur la semi-simplicité des produits tensoriels de représentations de groupes, Invent. Math., 116 (1994), 513-530, MR 94m:20091, Zbl 0816.20014.

[Tit79] J. Tits, Reductive groups over local fields, XXXIII (1), Proc. Sympos. Pure Math., Amer. Math. Soc., (1979), 29-69, MR 80h:20064, Zbl 0415.20035. 
Received October 26, 2000. This work was supported by a grant from the National Science Foundation.

Department of Mathematics

UNIVERSity OF Notre DAME

Notre DAme, IN 46556

E-mail address: McNinch.1@nd.edu 